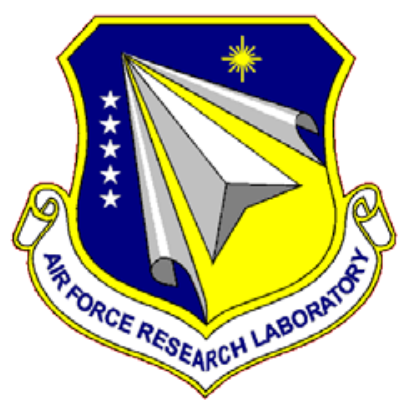

\title{
AFRL-RH-WP-TP-2014-0044
}

\section{Clinically Normal Stereopsis Does Not Ensure Performance Benefit from Stereoscopic 3D Depth Cues}

John P. McIntire*, Paul R. Havig, ${ }^{*}$ Lawrence K. Harrington**, Steven T. Wright***, Scott N. J. Watamaniuk****, and Eric L. Heft*

*USAF AFMC 711 HPW/RHCV, 2255 H St, B248 R300, Wright-Patterson AFB OH 45433-7022

**Ball Aerospace \& Technology Corp., 2875 Presidential Dr Ste 180, Fairborn OH 45324-6269

***USAF AFMC USAFSAM/FECO, $25105^{\text {th }}$ St, B840, Wright-Patterson AFB OH 45433-7913

****Wright State U., Psychology Dept, 3640 Col Glenn Hwy, 335 Fawcett Hall, Dayton OH 45435

October 2014

INTERIM REPORT

Distribution A: Approved for public release; distribution unlimited.

STINFO COPY

AIR FORCE RESEARCH LABORATORY

711 HUMAN PERFORMANCE WING

HUMAN EFFECTIVENESS DIRECTORATE

WRIGHT-PATTERSON AIR FORCE BASE OH 45433

AIR FORCE MATERIEL COMMAND

UNITED STATES AIR FORCE 


\section{NOTICE AND SIGNATURE PAGE}

Using Government drawings, specifications, or other data included in this document for any purpose other than Government procurement does not in any way obligate the U.S. Government. The fact that the Government formulated or supplied the drawings, specifications, or other data does not license the holder or any other person or corporation; or convey any rights or permission to manufacture, use, or sell any patented invention that may relate to them.

AFRL-RH-WP-TP-2014-0044 HAS BEEN REVIEWED AND IS APPROVED FOR PUBLICATION IN ACCORDANCE WITH ASSIGNED DISTRIBUTION STATEMENT.

//signed//

DARREL G. HOPPER

Program Manager

Battlespace Visualization Branch //signed//

JEFFREY L. CRAIG

Chief, Battlespace Visualization Branch

Warfighter Interface Division

//signed//

WILLIAM E. RUSSELL

Chief, Warfighter Interface Division

Human Effectiveness Directorate

711 Human Performance Wing

This report is published in the interest of scientific and technical information exchange, and its publication does not constitute the Government's approval or disapproval of its ideas or findings. 


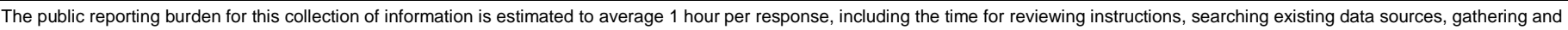

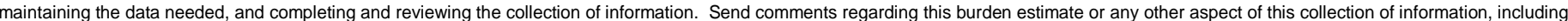

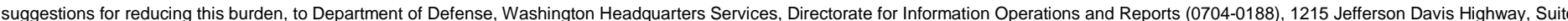

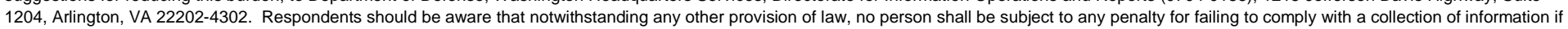
it does not display a currently valid OMB control number. PLEASE DO NOT RETURN YOUR FORM TO THE ABOVE ADDRESS

$$
\text { 28-10-2014 }
$$

Interim

1 May 2013 - 12 Mar 2014

\section{TITLE AND SUBTITLE}

\section{Clinically Normal Stereopsis Does Not Ensure Performance Benefit from Stereoscopic 3D Depth Cues}

\section{AUTHOR(S)}

John P. McIntire*, Paul R. Havig, *, Lawrence K. Harrington**, Steven T. Wright ${ }^{* * *}$, Scott N. J. Watamaniuk****, and Eric L. Heft*

\section{PERFORMING ORGANIZATION NAME(S) AND ADDRESS(ES)}

*USAF AFMC 711 HPW/RHCV, 2255 H St, B248 R300, Wright-Patterson AFB OH 45433-7022

**Ball Aerospace \& Technology Corp., 2875 Presidential Dr Ste 180, Fairborn OH 45324-6269

***USAF AFMC USAFSAM/FECO, $25105^{\text {th }}$ St, B840, Wright-Patterson AFB OH 45433-7913

****Wright State U., Psychology Dept, 3640 Col Glenn Hwy, 335 Fawcett Hall, Dayton OH 45435

9. SPONSORING/MONITORING AGENCY NAME(S) AND ADDRESS(ES)

Air Force Material Command

Air Force Research Laboratory

711 Human Performance Wing, Human Effectiveness Directorate

Warfighter Interface Division, Battlespace Visualization Branch

Wright-Patterson Air Force Base OH 45433-7022 5a. CONTRACT NUMBER

FA8650-08-D-6801-0050

5b. GRANT NUMBER

5c. PROGRAM ELEMENT NUMBER

Multiple

5d. PROJECT NUMBER

5239

5e. TASK NUMBER

11

5f. WORK UNIT NUMBER

H0CK (Historic: 53291102)

8. PERFORMING ORGANIZATION REPORT NUMBER

10. SPONSORING/MONITORING AGENCY ACRONYM(S)

USAF AFMC $711 \mathrm{HPW} / \mathrm{RHCV}$

11. SPONSORING/MONITORING AGENCY REPORT NUMBER(S)

AFRL-RH-WP-TP-2014-0044

\section{DISTRIBUTIONIAVAILABILITY STATEMENT}

Distribution A: Approved for public release; distribution unlimited.

13. SUPPLEMENTARY NOTES 88ABW Cleared 9/9/2013; 88ABW-2013-3986. Report contains color.

14. ABSTRACT To investigate the effect of manipulating disparity on task performance and viewing comfort, twelve participants were tested on a virtual object precision placement task while viewing a stereoscopic 3D (S3D) display. All participants had normal or corrected-to-normal visual acuity, passed the Titmus stereovision clinical test, and demonstrated normal binocular function, including phorias and binocular fusion ranges. Each participant completed six experimental sessions with different maximum binocular disparity limits. The results for ten of the twelve participants were generally as expected, demonstrating a large performance advantage when S3D cues were provided. The sessions with the larger disparity limits typically resulted in the best performance, and the sessions with no S3D cues the poorest performance. However, one participant demonstrated poorer performance in sessions with smaller disparity limits but improved performance in sessions with the larger disparity limits. Another participant's performance declined whenever any S3D cues were provided. Follow-up testing suggested that the phenomenon of pseudo-stereoanomaly may account for one viewer's atypical performance, while the phenomenon of stereoanomaly might account for the other. Overall, the results demonstrate that a subset of viewers with clinically normal binocular and stereoscopic vision may have difficulty performing depth-related tasks on S3D displays. The possibility of the vergenceaccommodation conflict contributing to individual performance differences is also discussed.

15. SUBJECT TERMS Stereopsis, Binocular Vision, Optometry, Depth Perception, 3D vision, 3D human factors, Stereoscopic displays, S3D, Virtual environment

16. SECURITY CLASSIFICATION OF:

\begin{tabular}{|l|l|l|} 
a. REPORT & b. ABSTRACT & c. THIS PAGE \\
Unclassified & Unclassified & Unclassified
\end{tabular}

17. LIMITATION OF ABSTRACT

SAR
18. NUMBER OF PAGES

26 19a. NAME OF RESPONSIBLE PERSON (Monitor) Darrel G. Hopper

19b. TELEPHONE NUMBER (Include Area Code) 


\title{
Technical Paper (TP)
}

\section{Clinically Normal Stereopsis Does Not Ensure a Performance Benefit from Stereoscopic 3D Depth Cues}

Reprint of paper submitted to: 3D Research (August 2014)

\author{
Authors: \\ John P. McIntire, PhD, 711 HPW/RHCV, Air Force Research Laboratory \\ Paul R. Havig, PhD, 711 HPW/RHCV, Air Force Research Laboratory \\ Lawrence K. Harrington, O.D., PhD, Ball Aerospace \& Technologies Corp. \\ Steven T. Wright, O.D., USAFSAM, Air Force Research Laboratory \\ Scott N. J. Watamaniuk, PhD, Psychology Department, Wright State University \\ Eric L. Heft, RHCV, 711 HPW/RHCV, Air Force Research Laboratory \\ Short Title: Individual Differences Using S3D for Depth-Related Tasks
}

\begin{abstract}
To investigate the effect of manipulating disparity on task performance and viewing comfort, twelve participants were tested on a virtual object precision placement task while viewing a stereoscopic 3D (S3D) display. All participants had normal or corrected-to-normal visual acuity, passed the Titmus stereovision clinical test, and demonstrated normal binocular function, including phorias and binocular fusion ranges. Each participant completed six experimental sessions with different maximum binocular disparity limits. The results for ten of the twelve participants were generally as expected, demonstrating a large performance advantage when S3D cues were provided. The sessions with the larger disparity limits typically resulted in the best performance, and the sessions with no S3D cues the poorest performance. However, one participant demonstrated poorer performance in sessions with smaller disparity limits but improved performance in sessions with the larger disparity limits. Another participant's performance declined whenever any S3D cues were provided. Follow-up testing suggested that the phenomenon of pseudo-stereoanomaly may account for one viewer's atypical performance, while the phenomenon of stereoanomaly might account for the other. Overall, the results demonstrate that a subset of viewers with clinically normal binocular and stereoscopic vision may have difficulty performing depth-related tasks on S3D displays. The possibility of the vergence-accommodation conflict contributing to individual performance differences is also discussed.
\end{abstract}

Key Words: Stereopsis, Binocular Vision, Optometry, Depth Perception, 3D vision, 3D human factors, Stereoscopic displays, S3D, Virtual environment 


\section{Introduction and Background}

Stereoscopic 3D (S3D) displays are surging in popularity and have found utility in many entertainment, engineering, medical, and military applications. Despite their wide popularity, and apparent ability to improve performance on depth-related tasks (McIntire, Havig, \& Geiselman, 2012, 2014), the majority of experimental evaluations of S3D displays present only grouped performance data overlooking idiosyncratic factors that may be of interest. If one were interested in operator selection criteria for a task that demanded color discrimination, there are a variety of optometric clinical color perception tests that can identify, with a high degree of confidence, who will perform well on such a task. Likewise, for a task requiring precise visual acuity, viewers with deficient vision can be easily identified.

But things are much more complicated for those interested in binocular vision and its relationship to stereoscopic depth task performance. There are a variety of clinical measurements and popular test booklets that can coarsely differentiate between viewers with "normal" stereopsis and those with reduced or deficient stereopsis, but the problem is that those classified as "normals" often do not behave as one might hope or expect when their task involves the viewing of a stereoscopic 3D display. This problem may be much more common than a naïve reader, unfamiliar with the literature, might be led to suspect. Specifically, in the vision science literature, there have been reports of two distinct but lesser-known phenomena that may have serious implications for individual performance on S3D display systems, because these may result in deficits for viewers that otherwise demonstrate clinically normal binocular and stereoscopic vision; these phenomena are referred to as stereoanomaly and pseudostereoanomaly. Individual differences in viewer's ability to modify the strength of their vergence-accommodation ( $\mathrm{V}-\mathrm{A}$ ) reflex, as is required for proper viewing of S3D stimuli, may also have implications for performance on S3D displays, though this is a more widelyrecognized problem. Relevant previous research on these three issues will be discussed next.

\section{Stereoanomaly}

Research by Richards $(1970,1971)$ demonstrated that as many as $30 \%$ of viewers with seemingly normal stereoscopic vision may have problems in perceiving certain types of stereoscopic disparity (i.e., crossed versus uncrossed). These stereoanomalous viewers only seemed to be identifiable when using briefly-presented stimuli with large (coarse) disparities in depth-discrimination tasks (Richards used 80 ms presentation times with a disparity range of 0.5 to $4.0 \mathrm{deg}$ ). Richards interpreted these stereoanomalies as possibly arising due to deficits in one or more neuronal "disparity pools" corresponding to crossed, uncrossed, and near-zero disparities.

Later research by Jones (1977) confirmed Richards' findings, showing a relationship between anomalous vergence eye movements and the phenomenon of stereoanomaly; the presence of vergence-anomaly was always associated with stereoanomaly (although not all stereoanomalous viewers demonstrated problems with vergence). Jones also confirmed that such anomalies could be present in viewers with clinically normal binocular vision and normal fine stereopsis, and suggested that the phenomenon of stereoanomaly was limited only to coarse stereopsis 
mechanisms. Like Richards, Jones used a large disparity range (0.6 to $4.6 \mathrm{deg}$ ) with brief presentation times (200 ms).

Richard's hypothesis that stereoanomaly arises due to deficits in specific disparity pools has since been in doubt due to more recent physiological, psychophysical, and computational modeling results, which instead suggest a continuous gradient in disparity-detecting mechanisms (e.g., Landers \& Cormack, 1997; Blake \& Wilson, 2011). But there remains ample physiological and psychophysical evidence for the existence of distinct neural processing streams underlying fine versus coarse stereopsis (e.g., Tyler, 1990). Fine stereopsis refers to small disparities (up to $\sim 20$ arc min) that are fused into a single binocular percept when presented briefly, and that result in increased perceptions of depth when disparity is scaled. Coarse stereopsis refers to larger disparities (>20 arc min) that are often reported as diplopic when presented briefly, even though a sensation of depth is elicited. However, the magnitude of perceived depth usually does not scale with increases in disparity for coarse stereopsis, and only a perception of "near" versus "far" seems apparent to the viewers.

In an experimental study, Shimono (1984) used a depth magnitude estimation task with disparities ranging from 0-50 arc min of crossed and uncrossed disparities, and presented the stimuli for $500 \mathrm{~ms}$. Two stereo-normal and three stereoanomalous viewers were tested (one stereoanomalous viewer had "non-normal" stereoacuity of 27 arc sec or worse). The results showed that the two stereoanomalous viewers with good stereoacuity had seemingly normal fine stereopsis, but deficient coarse stereopsis. Specifically, their reported depth magnitudes for large disparities were smaller than the stereo-normals, and large crossed disparities resulted in near/far confusions. The one stereoanomalous viewer with poor stereoacuity appeared to have deficits in both fine and coarse stereopsis. Shimono (1984) thus re-interpreted Richards' (1970/1971) results as a possible dysfunction in one or both of the separate processing streams underlying fine versus coarse stereopsis.

Research by Patterson and Fox (1984) again confirmed Richards' and others' findings of stereoanomaly with brief stimuli, but found that when longer viewing periods were permitted, most subjects who were classifiable as stereoanomalous performed normally. They suggested that previous reports of stereoanomaly may be due to quirks in the particular testing method employed, or duration effects, instead of indicating deficits in underlying neural mechanisms. Landers and Cormack (1997) discovered that most subjects made faster and more accurate depth judgments for crossed disparities, and their thorough review of the issue suggested the possibility that stereoanomaly may be due to errors in disparity sign computation (crossed versus uncrossed) that is dissociable from computations of disparity magnitude or disparity detection. This is a possibility we find particularly intriguing.

It should be noted that van Ee and Richards (2002) and van Ee (2003) have challenged these alternative interpretations, and provided several additional lines of evidence in support of stereoanomalies being due to deficits in crossed versus uncrossed disparity mechanisms, including: evidence from neurophysiology; different developmental rates in infants for crossed and uncrossed disparities; and in adults, different spatiotemporal response functions for the two types of disparity (a more complete though older review of this evidence is provided by Mustillo, 1985). Kooi, Dekker, van Ee, and Brouwer (2010) proved that stereoanomaly is a robust 
phenomenon that occurs even in depth-tasks using real-world true 3D stimuli, and is not limited only to S3D displays in which depth cue conflicts are present (the vergence-accommodation conflict will be discussed in a later section).

The question of what exactly is stereoanomaly does not seem to have been convincingly settled, but the current thinking is that stereoanomaly may be due to differences or deficits in fine versus coarse stereopsis mechanisms, or perhaps in the computations of disparity sign versus disparity magnitude versus disparity detection, but often manifesting as differences between crossed and uncrossed disparity judgments. And stereoanomaly is associated in some viewers with vergenceanomaly. It is worth noting here that in the literature, the phenomena of stereoanomaly appears to be limited to briefly presented stimuli, but this issue too will be addressed in later sections.

\section{Pseudo-Stereoanomaly}

Kihara, Fujisaki, Ohtsuka, Miyao, Shimamura, Arai, and Taniguchi (2013) conducted a depth magnitude estimation task using combinations of monocular and binocular cues to depth. Three levels of shading and three levels of crossed disparity $(0,4$, and 8 arc min) were tested across a sample 134 viewers of various ages. All included participants were classified as having "normal" stereoscopic vision according to the Titmus (Stereo Fly SO-001) clinical stereotest which indicated better than 4 arc min of stereoacuity for all participants. It should be noted here that standard clinical practice usually requires much better stereoacuities of 30-40 arc sec or lower (in this case, six times better) to be considered normal (e.g., Fielder \& Moseley, 1996). In any case, Kihara et al. (2013) discovered that participants tended to fall into one of four classes: (1) A cuecombination group who utilized both types of depth cues to perform their depth judgments (41.8\%); (2) a disparity-cue group who seemed to utilize only binocular disparity cues to perform the task (16.4\%); (3) a pictorial-cue group who seemed to utilize only monocular pictorial cues (32.8\%); and (4) a low-depth perception group who reported the stimuli as appearing flat regardless of stimulus condition (9.0\%).

The researchers interpreted these individual differences in depth perception performance as being due to viewers' unique "strategies” or weightings for the various depth cues affecting how the cues are combined into a single coherent depth percept of objects in a scene. In other words, individual viewers seem to differentially favor some depth cues over others. Kihara et al. (2013) referred to the pictorial-cue group as possessing pseudo-anomalous stereopsis since these viewers did not utilize the disparity cue information in their depth judgments, even though they could apparently see S3D cues (on the Titmus test). They also hypothesized that this pseudostereoanomalous group would abandon their cue-weighting strategy, and utilize stereopsis cues, for tasks in which extremely accurate depth judgments are required (as opposed to the rough depth estimation required for their task), since binocular disparity gives higher fidelity depth information relative to pictorial cues (p. 502). A similar experiment by the same group using only young participants (19-25 years old) with no stereoanomalies also found about 30\% of viewers fell into the pictorial-cue group, apparently not utilizing disparity cues to aid depth judgments (Fujisaki, Yamashita, Kihara, \& Ohtsuka, 2012).

Vergence-accommodation Conflict and S3D Displays 
Individual differences in viewers' ability to modify the V-A reflex ("stretch" the link between vergence and accommodation) for S3D stimuli may have implications for performance. This may be especially pertinent since larger disparities (larger V-A conflicts) have already been clearly demonstrated to contribute to eyestrain and fatigue effects from viewing S3D displays, and to affect performance on rapid visual tasks like speeded identification of a stereoscopic stimulus and time-limited stereoacuity measures (e.g., Hoffman, Girshick, Akeley, \& Banks, 2008). Whether the V-A conflict contributes directly to more complex performance tasks on S3D displays remains uncertain but likely, given that the inability to modify the V-A link in the presence of $\mathrm{V}$-A conflict could result in diplopic, unfused stimuli, and thus result in degraded depth perception. An illustration of the V-A conflict caused by S3D displays is presented in Figure 1.

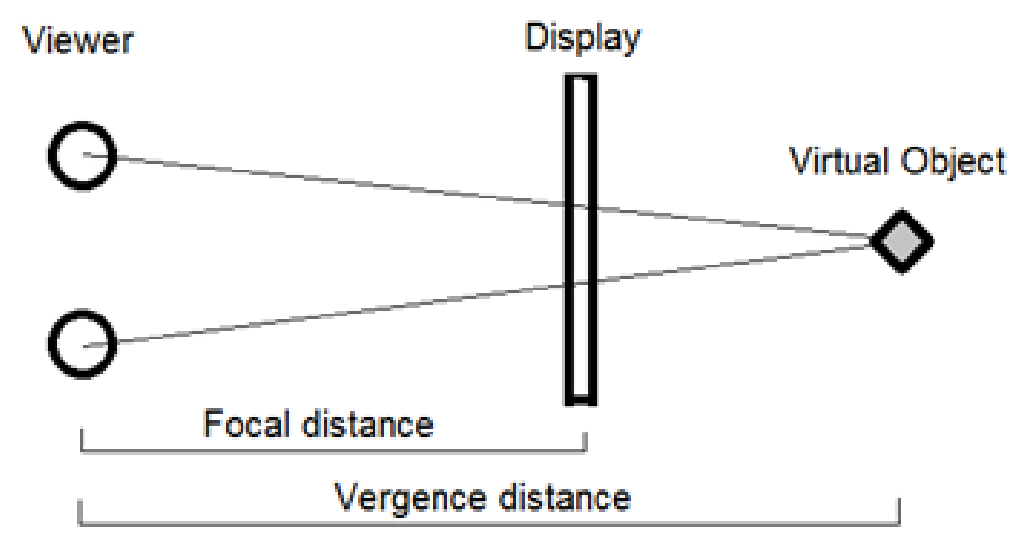

Figure 1. An illustration of the Vergence-Accommodation (VA) conflict. On a stereoscopic 3D display, the depth of a virtual object requires vergence eye movements for a particular distance that is different from the focal distance of the display, creating conflict between the two neurologically-linked systems underlying accommodation and vergence.

Hoffman, Girshick, Akeley, and Banks (2008) also noted in their discussion (p. 21) that they had to exclude a large number of potential subjects from their experiment because the volunteers could not comfortably and/or effectively fuse stereoscopic stimuli with large V-A conflicts. This is despite the fact that the excluded volunteers had passed the Titmus stereovision clinical test, verifying stereoscopic acuity of $\sim 40$ arc sec or better, and could effectively view binocular stimuli in the real-world over a wide range of distances. Similarly, Froner (2011) conducted two depth detection tasks in which the participants were required to identify which one of two squares was in depth (with crossed disparity) relative to the display surface. The author noticed that some individuals performed more poorly than would be expected based upon their visual acuity (20/20 or better) and stereoscopic acuity ( $\sim 40$ arc sec or better) pre-screening test results. Froner speculated that V-A conflict issues may have been a contributing factor that degraded performance for those observers (p. 193). 


\section{The Present Research}

The phenomena of stereoanomaly and pseudo-stereoanomaly, and the problematic issue of V-A conflict inherent in S3D displays, suggest there may be significant individual differences in stereoscopic depth perception capabilities when viewing S3D. There is concern that such problems may not be easily detectable for any given individual viewer using standard clinical optometric measures, which may have serious implications for performance on S3D display systems in applied settings. This is especially true if, in the future, normal stereo vision is a requirement for certain jobs involving the use of S3D displays, in the same way that police officers and pilots are screened for acuity and color vision.

In this work, we describe an experiment using an S3D display for a virtual object precision placement task, in which we originally set out to study the effects of using different levels of virtual camera separation (i.e., binocular disparity limits) on performance and comfort. However, during the course of our research, we made the happenstance discovery that all three issues of stereoanomaly, pseudo-stereoanomaly, and V-A conflicts may play a key role in accounting for some of the unexpected individual differences in performance that we observed in our data. This individualized, anomalous data is what we focus on in the present report. For interested readers, some different analyses of performance, comfort, and optometric measurement data from this and a related experiment are reported in detail in McIntire, Wright, Harrington, Havig, Watamaniuk, \& Heft, (2014) and McIntire, Wright, Harrington, Havig, Watamaniuk, \& Heft (in preparation). 


\section{Methods}

Participants. This study was approved in advance by the Air Force Research Laboratory's (AFRL) Institutional Review Board (IRB) and classified as minimal risk. Each participant provided signed consent before participating. Twelve participants were included in this study, ranging in age from 19 to 35 years old, with a mean age of 28 years. The male-to-female ratio was 7:5. All had normal or corrected-to-normal distant visual acuity in each eye (20/20 or better) and scored near-perfect on the Titmus stereovision test (indicating near stereoacuity of 40 arc sec or better). Normal binocular function was confirmed through measurement of distant and near phorias and vergence ranges. Additionally, subjects were screened for suppression tendencies using the AO Suppression test. Two volunteers were excluded due to abnormal findings on this battery. A brief demographic and personal history questionnaire related to S3D was also administered, and inter-pupillary distances (IPDs) were measured by the experimenter.

Display and Apparatus. A high-resolution temporally-multiplexed $120 \mathrm{~Hz}$ stereoscopic 3D display was used to present the imagery to the participants (NVIDIA Personal GeForce 3D Vision Active Shutter Glasses, and Samsung SyncMaster 2233RZ). This display was a 22-inch diagonal LCD display with a refresh rate of $120 \mathrm{~Hz}$ with native resolution of 1680 (horizontal) $\mathrm{x}$ 1050 (vertical), shown in Figure 2. This display system required the wearing of electro-optical active shutter glasses that rapidly oscillated between translucence and opacity in synchrony with the display's oscillation between each eye's imagery (60 Hz per eye). For the purpose of this study, observers viewed this display at a distance of approximately 24 inches. A standard QWERTY keyboard and mouse were utilized for the participants' interactions with the display system.

Software. A Microsoft Excel workbook was created to track each participant's progress through the randomized ordering of pre and post-tests. The primary task was written in Visual C++ using the OpenSceneGraph library to handle creation and manipulation of the viewing volume on the stereoscopic display. Care was taken to match the screen size and viewing distance to the virtual camera and viewing volume. The disparity calculations were verified by placing the test object at a series of distances into and out of the screen, and a high resolution camera captured the resulting left-right image pairs, allowing for on-screen disparities (stereopair half-image separations) to be measured.

Task. The task required the precision placement (spatial alignment) of a virtual object. For each trial, the participant used their right hand to control a computer mouse to position a virtual object (e.g., a small textured pyramid or peg, the "control” object) at an indicated depth on the display, matching the depth and vertical positioning of a reference or "target" object. This task served as a replication-and-extension of previous work by Rosenberg (1993) who tested a similar virtual object positioning task and measured alignment accuracy. On each trial, the target object appeared at a randomly chosen point on the target plane. The control object started every trial at the intersection of the control plane and the screen plane, centered along the $x$-axis. Movement of the control object was limited to the horizontal $(\mathrm{x}-\mathrm{z})$ control plane. The target object remained stationary at all times during each trial. 


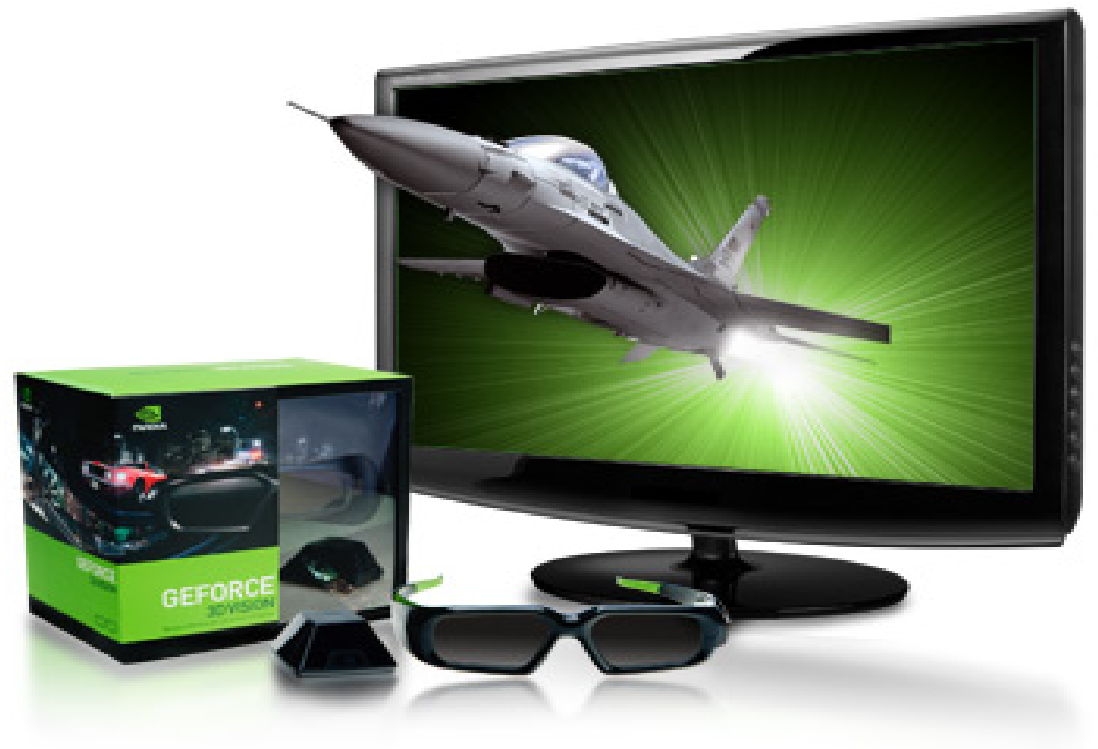

Figure 2. The S3D display with wireless active shutter glasses used in the present experiment. The display was a 22-inch diagonal $120 \mathrm{~Hz}$ LCD, with a resolution of $1680 \mathrm{x} 1050$. Image adapted from Samsung Syncmaster and NVidia GeForce promotional material.

The following magnitudes of the computer-generated stimuli are reported in virtual inches, as the computer model of the task was designed to correspond as accurately as possible with the realworld viewer/display space. The target and control planes were vertically separated by a gap of 2 inches, and measured 8 inches wide by 14 inches deep. The two planes both extended in the zdimension of virtual space 5.1 inches coming out of the screen, towards the viewer, and 8.8 inches behind the screen away from the viewer. Both the target and control objects were 1.0 inch tall and 0.56 inch at their widest, and centered vertically in their respective planes, so the vertical separation between the bottom of the control object and the top of the target object was 1 inch. See Figures 3 and 4.

Participants pressed the keyboard space bar with their left hand when satisfied with the alignment. Performance measures included completion times and positional error (difference between optimal placement and actual placement in $\mathrm{x}-\mathrm{z}$ space). Accuracy was emphasized as the primary measure of interest.

Stimuli \& Disparities. Binocular disparity limits (also known as “disparity brackets”, “disparity ranges”, or “depth budgets”; e.g., see Kytö, Hakala, Oittinen, \& Häkkinen, 2012) were fixed within each session to limit the amount of disparity (crossed or uncrossed) on any given trial to a maximum of $0,20,40,60,80$, or 100 arc min. This manipulation was analogous to fixing virtual camera separation in each session to a single value, which differed across sessions. Another 


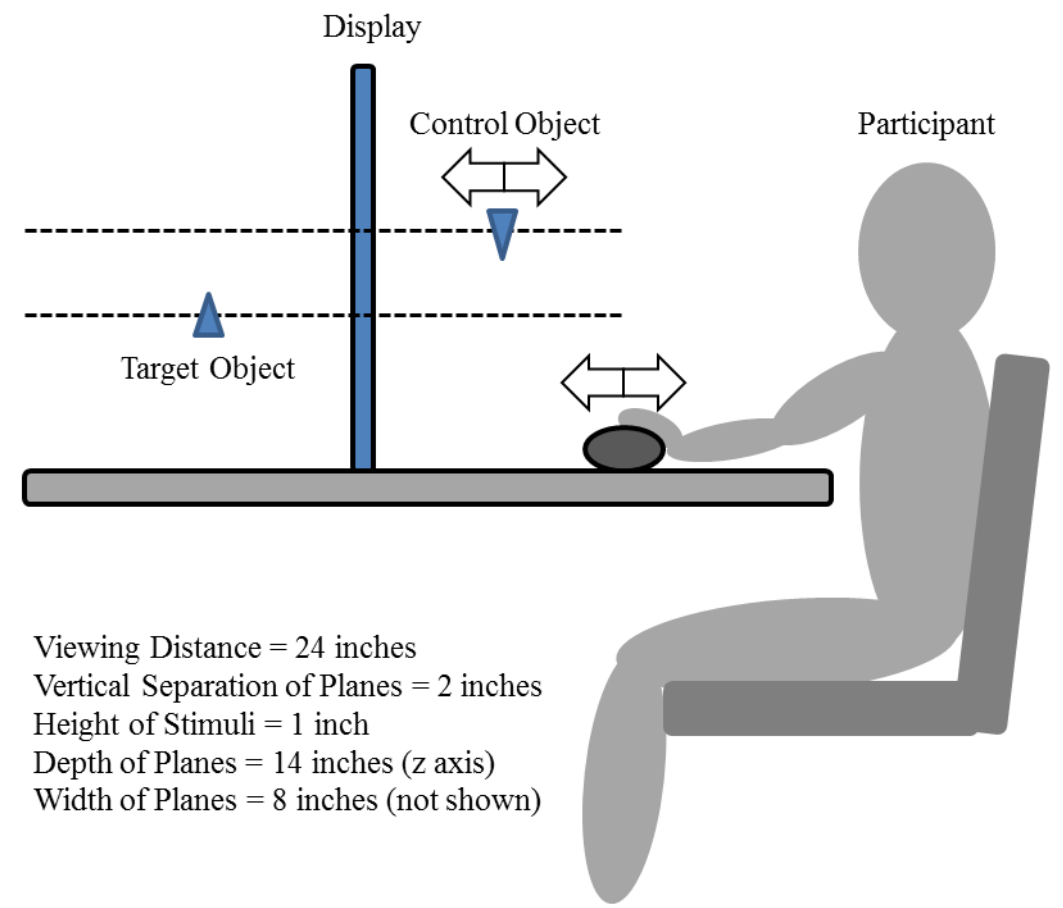

Figure 3. Schematic side view of the experimental set-up. The participant physically controlled a computer mouse to move the control object within the virtual volume, presented to the viewer via the S3D display. Movement of the control object was limited to the control plane. The task required the precise alignment of the control object overtop the target object.
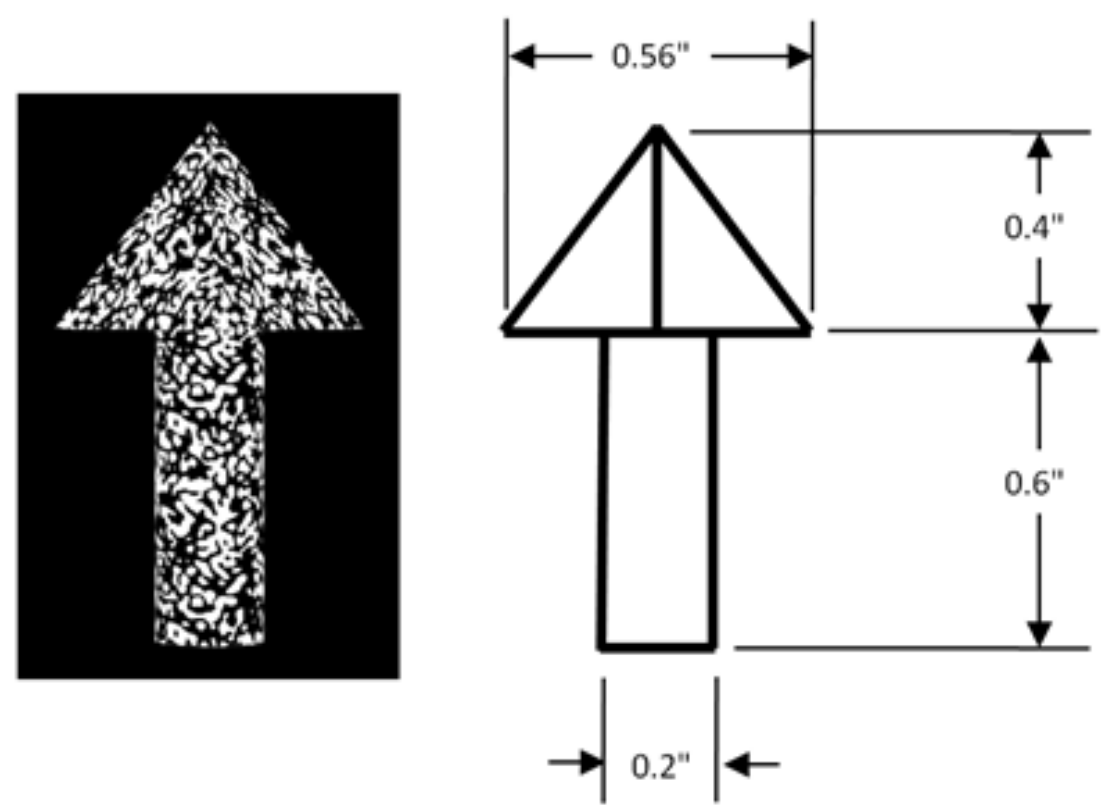

Figure 4. A screenshot (left) and schematic (right) of the virtual objects that are used in the virtual object alignment task. 
analogous way to think about this manipulation is that the virtual IPD ranged from 0 to $100 \%$ (assuming an average IPD of 2.6 inches, or $66 \mathrm{~mm}$ ) in 20\% steps of "microstereopsis", with $0 \%$ corresponding to a session with no stereopsis cues, and $100 \%$ corresponding to sessions with orthostereoscopic disparity cues. See the Table 1 for comparisons between these equivalent formulations. Each experimental session presented only one limit/range per session. The order in which disparity limits were presented (one per session) was randomized across participants via a Latin Square design.

Procedure. Before each experimental session, participants' horizontal phorias (near and far) and vertical phoria (far) were measured using the Keystone View Telebinocular vision screening apparatus (Keystone View Company/Mast Development Company; Meadville, Pennsylvania). We also measured participants' fusion ranges for stereoscopic stimuli on the S3D display using a modified method of adjustment. A stimulus at the plane of the display was slowly moved inward using crossed disparity (towards the viewer in depth) until the image either became blurry or broke into a double image, at which point the viewer signaled with a button press. The image was reset at the depth plane of the screen and moved in the opposite direction, again until the image either became blurry or broke into two, and again the viewer signaled this event with a button press. This gave measures of the near and far points of fusion (the zone of clear, single binocular vision). The distance between these two points is the fusion range.

After these brief pre-testing measurements, the 30-minute experimental session began. Trials were entirely self-paced. A total of six sessions (corresponding to the six disparity limit manipulations) were completed by each participant. Each experimental session was completed on a different day. Five-minutes of practice/training were permitted before the start of the first session. Participants on average completed 300 trials per session with an average response time of 6 seconds per trial.

Table 1. Equivalent formulations of the disparity limits used in the experiment (one limit per session). The binocular disparity limit manipulation can also be considered as manipulation in virtual camera separations, either in raw distance units $(\mathrm{mm})$ or in terms of percentage of a virtual IPD (percentage).

\begin{tabular}{lcccccc} 
Stereopsis Cues : & none & \multicolumn{3}{c}{ micro-stereopsis } & & ortho \\
$\begin{array}{l}\text { Binocular Disparity } \\
\text { Limit (arc min) }\end{array}$ & 0 & \pm 20 & \pm 40 & \pm 60 & \pm 80 & \pm 100 \\
$\begin{array}{l}\text { Virtual camera } \\
\text { separation (vIPD\%) }\end{array}$ & 0 & 20 & 40 & 60 & 80 & 100 \\
$\begin{array}{l}\text { Virtual camera } \\
\text { separation (mm) }\end{array}$ & 0.0 & 13.2 & 26.4 & 39.6 & 52.8 & 66.0 \\
& & & & & &
\end{tabular}




\section{Results}

Across participants, there was a large benefit to performance by providing disparity cues to depth with an average reduction of 53\% in mean placement error magnitudes when compared to the zero-disparity trials. For most participants, providing disparity cues greatly improved placement accuracy: half of the participants reduced their mean placement error by $70 \%$ or more. But there were notable individual differences in performance. We identified two sub-groups ("typical" and "atypical") of observers based upon the benefit they received from disparity cues. Table 2 lists the performance of the twelve participants, and makes clear that Participants 7 and 10 have atypical performance when presented with disparity cues (on average, showing no benefit, or a performance detriment).

Table 2. Mean placement error (in virtual inches) for each participant in both the no-stereo and S3D display conditions, and the percentage improvement when changing from no-stereo to S3D conditions. Each reported value for S3D represents the mean of all trials in which any magnitude of disparity was present. The shaded rows indicate the participants with atypical performance.

\begin{tabular}{|c|c|c|c|}
\hline Participant & $\begin{array}{c}\text { Non-stereo Display } \\
\text { Mean Placement Error } \\
\text { (virtual inches) }\end{array}$ & $\begin{array}{c}\text { S3D Display } \\
\text { Mean Placement Error } \\
\text { (virtual inches) }\end{array}$ & $\begin{array}{c}\text { \% Reduction in } \\
\text { Errors with S3D Cues }\end{array}$ \\
\hline 1 & 1.50 & 0.30 & 80 \\
\hline 2 & 1.19 & 0.30 & 74 \\
\hline 3 & 0.97 & 0.28 & 80 \\
\hline 4 & 1.49 & 0.30 & 30 \\
\hline 5 & 0.94 & 0.66 & -22 \\
\hline 6 & 1.32 & 0.65 & 55 \\
\hline 7 & 0.96 & 1.16 & 83 \\
\hline 8 & 0.75 & 0.34 & 1 \\
\hline 9 & 1.34 & 0.22 & 91 \\
\hline 10 & 0.95 & 0.94 & 42 \\
\hline 11 & 2.04 & 0.19 & $\mathbf{5 3}$ \\
\hline 12 & 0.84 & $\mathbf{0 . 4 9}$ & \\
\hline Average & $\mathbf{1 . 1 9}$ & & \\
\hline
\end{tabular}


As shown in Figure 5, the results from the typical subgroup (10 of 12 participants) show what we might expect to see if individuals generally benefited from S3D cues, in which increasing disparity limits positively improved depth task performance. This subgroup demonstrates a huge benefit with stereo 3D, ranging from $30 \%$ to $91 \%$ reductions in placement errors when provided with disparity cues. Although participants 5 and 12 show a non-monotonic pattern, on average they both experienced a benefit from disparity cues (30\% and $42 \%$ average reduction in errors, respectively) and generally obtained larger benefits in sessions with larger disparity limits. Some participants' performances either peaked or plateaued at 60 or 80 arc min of disparity, suggesting no further benefit from enlarging disparities beyond these limits. Interestingly, several participants in this subgroup never reached a clear asymptote, and might have continued to benefit from disparities limits beyond the range tested (up to $100 \mathrm{arc}$ min). We would expect all participants' performance curves, if given a large enough range of disparities, to eventually asymptote due to reaching and then exceeding the limits for binocular fusion which are individual specific (e.g., Rozhkova \& Vasiljeva, 2010). A fusion break results in diplopic and/or blurry vision, which can result in binocular rivalry with monocular suppression, viewing discomfort, and/or associated visual problems; ultimately, performance decrements on depthrelated tasks would appear. 

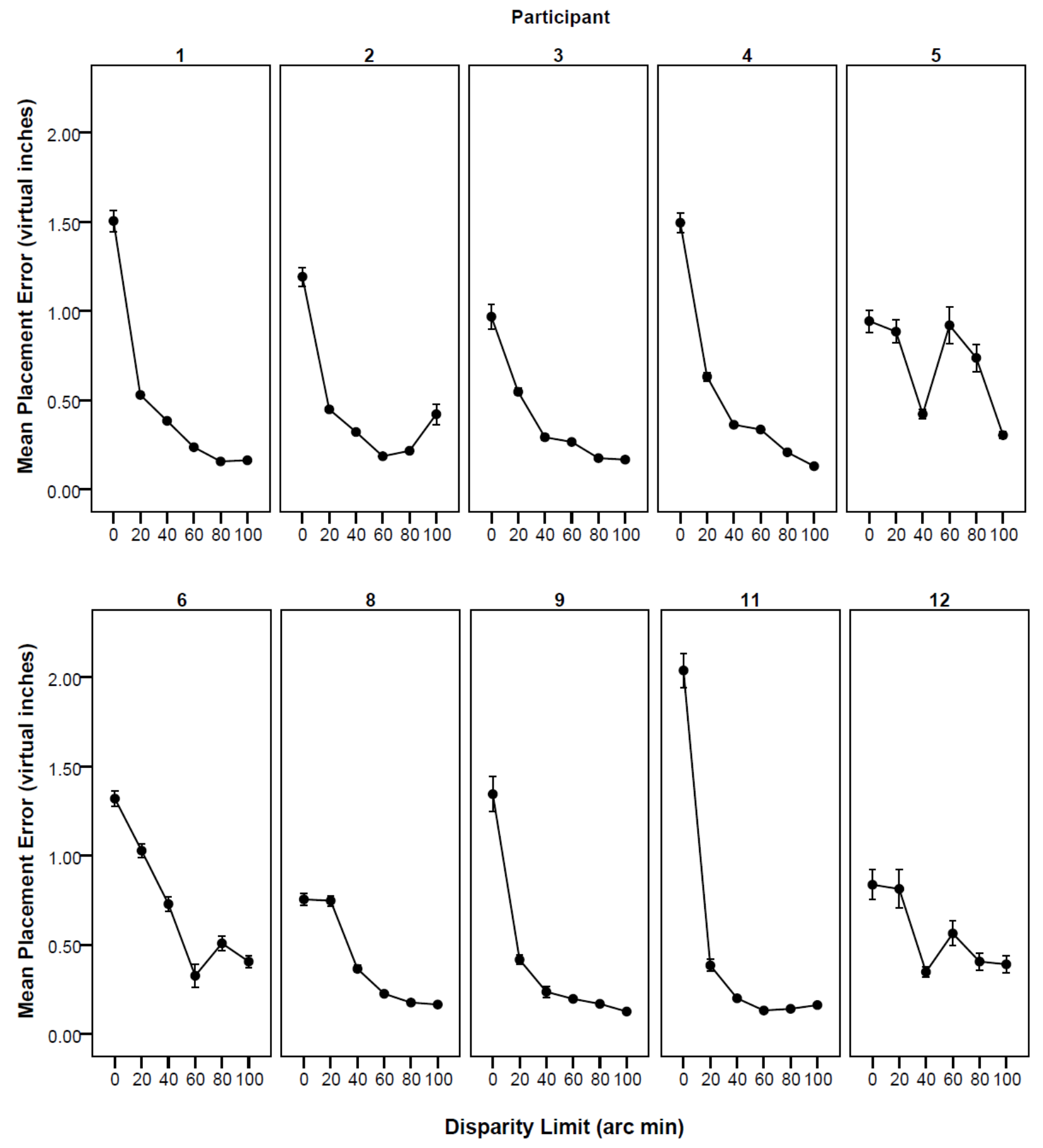

Figure 5. The effect of binocular disparity limit on mean placement error for individual participants. These data represent a majority subgroup ('typical') that clearly benefited from having disparity cues. Error bars represent +/- 1 SEM; when not visible, error bars are smaller than the data symbol. 
Two participants demonstrated an "atypical” pattern of results, as shown in Figure 6. Comparing the performance curves in Figure 6 to those in Figure 5 exemplifies the uniqueness of their performance relative to the "typical" performers (the average differences are also notable in Table 2). Participant 10 on average received no benefit from the S3D cues; but interestingly, performance was enhanced by the large disparity limits while performance was worse than the zero-disparity condition with the small disparity limits. Participant 7 received absolutely no benefit from S3D disparity cues, and appeared to perform more poorly as disparity limits were increased above zero.

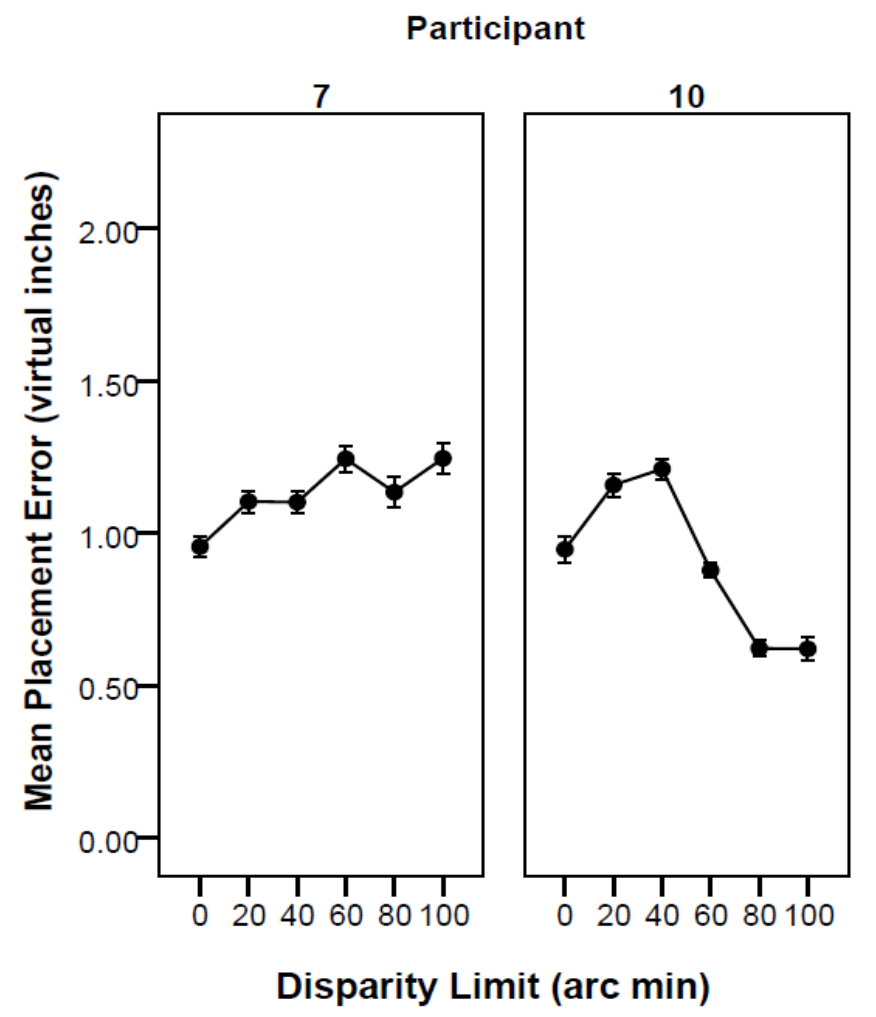

Figure 6. The effect of binocular disparity limit on mean placement error for the minority subgroup of participants whose benefit from S3D disparity cues was overall neutral (Participant 10) or negative (Participant 7). Error bars represent +/- 1 SEM; when not visible, error bars are smaller than the data symbol. 


\section{Discussion and Follow-up Testing}

Recall that all participants tested normal on the Titmus stereovision clinical test and demonstrated normal binocular function on optometric screening. This is a potentially disturbing finding for real-world applications of S3D because it suggests the possibility that some viewers with normal clinical findings might fail to perceive 3D stimuli as intended and could result in performance comparable to 2D (with no benefit of S3D disparity cues); or even worse, performance may be impaired by S3D disparity cues. These unexpected results motivated us to look more closely at the observers with 'atypical' performance and to conduct follow-up research, after we verified that the 3D shutter glasses were functioning normally.

Perusal of the demographic and personal history questionnaires, involving questions of history of viewing 3D movies/games/TV, age, gender, inter-pupillary distance, experience of migraines, motion sickness, et cetera revealed no obvious explanations as to why these two participants would have little or no benefit from S3D. We should also note that the two individuals were not noticeable outliers in any of the optometric screening tests.

The two participants with atypical performance reported being able to see "3D" and "depth" though perhaps not a strong sensation of it. For instance, Participant 10 noted in a follow-up conversation: "Now that I think about it, when starting a new session I never thought to myself, 'Oh this is definitely 2D or 3D.' I only knew for sure it was 3D when I saw double." This reference to double vision could have referred either to diplopia due to loss of fusion from large disparities, or it could refer to the subtle interocular crosstalk inherent in the S3D display system in which images intended for one eye "bleed through" to the unintended eye, which perceptually can result in a dim doubling or tripling of the stimulus, even when fusion is occurring as intended. However, mild magnitudes of crosstalk do not typically induce discomfort (e.g., Kooi \& Toet, 2004) nor would we expect it to impact performance, unless the crosstalk interfered with the fusional process (by creating false binocular matches) or perhaps by serving as an inadvertent cue to position in depth (by allowing a viewer to align the ghost images of the target with the ghost images of the control object). If this latter explanation were correct, though, we would expect performance to have improved with the larger disparities since the ghost image separations would be more obvious and allow for more precise alignment (this may explain the performance of Participant 10, but not that of Participant 7).

It is worth noting that these participants had generally fast response times, though they were not the fastest, and had relatively good performance in the zero-disparity (no stereopsis) condition, though they were not the best. Both participants denied having inadvertently closed one eye due to any visual discomfort induced by the S3D display itself, or due to the glasses. Little or no discomfort was induced by the display, according to these participants' self-reports and the SSQ ratings; indeed we might expect viewers who are not perceiving the S3D effect to find the display perfectly comfortable since there would be no V-A conflict contributing to fatigue/eyestrain.

These participants with atypical performance may have traded-off accuracy for speed in an attempt to "rush through" the study (even though it was time-limited to 30 minutes regardless of speed) or due to misunderstanding the instruction to focus on accuracy as the more important 
measure. These possibilities seem unlikely from an examination of their response time data, as do training or practice effects.

Type of Displayed Disparity

Further examination of the performance data was conducted. We plotted performance in terms of the type of displayed disparity (crossed, uncrossed, or planar) versus performance in the zerodisparity session. Note that here "type of disparity" refers to the direction of binocular disparity of the target object on a given trial relative to the display surface, and "planar" refers to the location of the virtual target object being centered at the plane of the display surface. In other words, stimuli with "crossed" disparity have negative image parallax and require convergent eye alignment (crossing the eyes) relative to the display surface. Alternatively, "uncrossed" disparity has positive image parallax and requires divergent eye movements (uncrossing the eyes) for binocular fusion. And "planar" requires no additional vergence eye movements off the plane of the display surface (zero image parallax).

When performance was plotted in this way, the pattern of results was different between the two 'atypical' individuals, and both were distinctly different than any of the other individuals' patterns which all appeared very similar to each other (see Figure 4). In general, participants received a large beneficial effect of disparity cues, regardless of disparity type, as expected. Also, uncrossed disparity usually resulted in slightly less accurate performance than crossed or planar disparities, though the magnitude of difference was relatively small across participants.
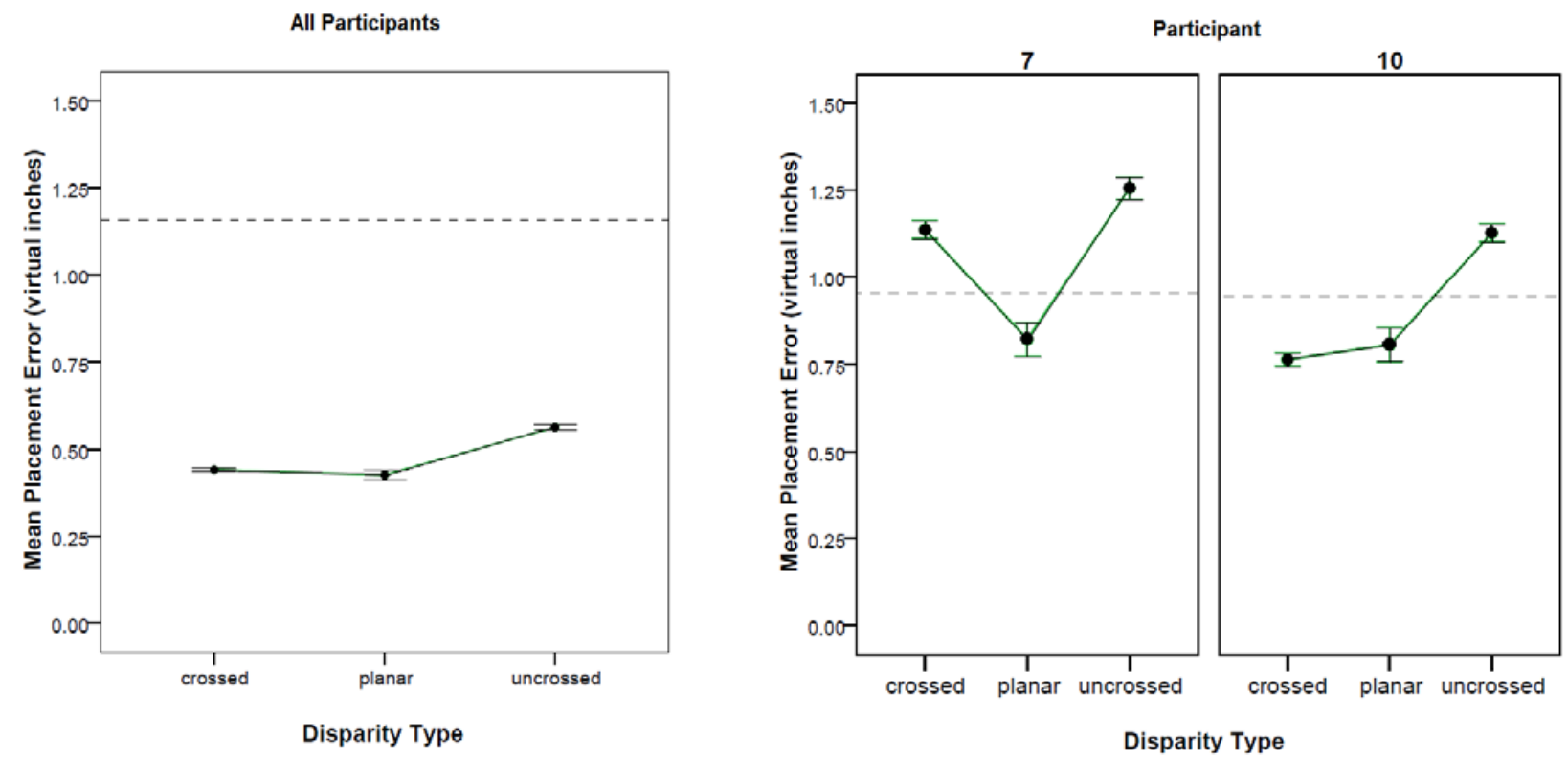

Figure 7. The effect of S3D disparity type (crossed, uncrossed, or planar) on placement errors, compared to performance in the session(s) with no disparity cues. The left panel shows data averaged across all participants (including 7 and 10) which demonstrates the typical pattern. The panels on the right show the results for the two participants with atypical performance. The dashed lines represent the corresponding mean performance in the zero-disparity session(s). Error bars represent +/- 1 SEM. 
Participant 7 seemed to slightly benefit from S3D disparity cues, but only if the target object was portrayed at the surface of the display (planar); otherwise, crossed or uncrossed disparities hurt performance relative to the 2D condition. Perhaps even stranger, for Participant 10, disparity cues also slightly helped performance, but only for crossed or planar disparities. For both participants, uncrossed disparities resulted in performance worse than their zero-disparity baseline.

These data suggest that these two participants with atypical performance apparently perceived some stereoscopic depth from the 3D display, but only if the virtual object was located at the surface of the screen in depth. However, even if they were seeing some stereoscopic depth, the beneficial effect was mild for both viewers, and transformed into a hindrance with either uncrossed disparity (Participant 10) or both crossed and uncrossed disparities (Participant 7). At first glance, these results might seem to confirm the existence of different forms of stereoanomaly (e.g., Richards, 1970/1971) for both participants. If true, this would be the first description of the phenomenon of stereoanomaly in performance data involving complex dynamic interaction with virtual objects over extended viewing periods, as opposed to using a depth-detection or depth-magnitude-estimation task with briefly presented stimuli. Participant 7 might even be explainable in terms of deficiencies in course versus fine stereopsis mechanisms, as was suggested for stereoanomaly by Jones (1977) and Shimono (1984). These results may also be attributable to deficiencies in making the proper vergence eye movements to stereoscopic stimuli (particularly for uncrossed locations), and perhaps an inability to effectively keep the eyes in alignment at the appropriate depth. A relationship between eye movements and stereoanomaly was explored by Jones (1977), as mentioned previously, who found that vergence-anomalous viewers always demonstrated stereoanomaly, but not vice versa. 


\section{Follow-up Data Collection}

Some additional brief follow-up tests were conducted with the two participants who demonstrated atypical performance, and stereoacuities were also measured for all participants on a custom thresholding task presented on an S3D display. The stereoacuity thresholding task involved 50 trials of "near" versus "far" judgments of a small virtual target rod flanked by two reference rods, situated at the plane of the display. The psychophysical technique employed the adaptive QUEST method (Watson \& Pelli, 1983). We also conducted a modified virtual object precision placement task in which 50 trials contained no disparity cues, followed by 50 trials containing orthostereoscopic disparity cues (disparity limit of \pm 100 arc min). In all trials we randomly manipulated the sizes of the virtual objects so that monocular (pictorial) cues were unreliable, and thus unhelpful for the placement task. The idea was to force the use of the S3D disparity cues, in case the atypical performers were simply choosing to ignore (or loosely weight) the stereoscopic cues to depth.

Participant 10: When forced to use binocular disparity cues on the modified placement task, error magnitudes decreased in the S3D condition by 83\% (from an average of 6.0 to 1.0 inches) and the standard deviation of errors shrank $75 \%$ (from 3.7 to 0.9 inches) while response times remained unchanged. For this participant, the stereoacuity threshold was measured at 26 arc sec and additional optometric screening demonstrated no evidence of suppression or binocular disorder.

Participant 7: When forced to use binocular disparity cues for the modified placement task, error magnitudes increased in the S3D condition by 5\% (from an average of 4.1 to 4.3 inches), the standard deviation of errors also increased by 52\% (from 2.9 to 4.4 inches), and response times were lengthier. It seems clear that this participant was simply unable to see stereoscopically on the S3D display as intended, and reported great difficulty in performing the requested tasks when taking the threshold measurement and on the forced binocularity placement task. Participant 7's stereoacuity was retested at 25-30 arc sec using the Optec Vision Tester (OVT). Further binocular testing also demonstrated no evidence of binocular dysfunction or suppression tendencies.

It should be noted that these stereoacuity threshold values in the range of 30 arc sec are typically classified as clinically normal, but are not optimal. Under ideal conditions, in the best observers, thresholds of 2-3 arc sec are obtainable (Fielder \& Moseley, 1996). It should also be pointed out that the stereoacuities of the two participants with atypical performance were the worst out of the twelve participants. The average of the other ten participants' stereoacuity thresholds was at least twice as good at about 12 arc sec. 


\section{General Discussion}

In summary, in our attempts to determine the cause of the two viewers' atypical performance with follow-up testing, we can say with confidence: (1) both observers had at best stereoscopic acuities of around 25-30 arc sec, so they should have been easily able to perceive and utilize the disparity cues used in the present study (up to \pm 100 arc min); (2) neither observer indicated any sort of monocular suppression occurring; (3) one participant was able to use disparity cues to improve performance when forced (Participant 10), while the other (Participant 7) apparently could not properly perceive the stimuli on the S3D displays either on the forced 3D task or on the stereoacuity threshold task when administered on an S3D display.

This leaves us with two different probable (though not definitive) explanations. Participant 10's atypical performance seems to be due to a different unconscious "strategy" or "depth cue weighting” being deployed in the performance of the S3D placement task. This participant appears to have been more heavily weighting the monocular pictorial cues of size and/or texture over the disparity cues to depth of the objects. Apparently, when the disparity cues were large enough (80 or 100 arc min), this seemed to allow disparity to finally help, perhaps by exceeding some threshold of activity in the disparity signaling mechanism that overtook the signaling of pictorial cues. When forced to use only S3D cues in the follow-up modified task, the participant was able to do so effectively to improve performance over the zero-disparity trials.

This interpretation seems consistent with the results of Kihara et al. (2013), who demonstrated individual differences in how viewers utilize and combine various cues in a scene to determine depths of objects, which they described as pseudo-stereoanomaly. If true, the present results may be the first demonstration that individual differences in depth cue weightings affect not only depth judgments in a small disparity range (as in Kihara et al., 2013), but also performance on an active depth placement task across a larger disparity range. And that such performance differences may manifest even in a sample of observers with clinically normal stereopsis and with good stereoacuity.

Kihara et al. (2013) had hypothesized that pseudo-stereoanomalous viewers would abandon their pictorial-cue-weighting strategy, and utilize stereopsis cues, for tasks in which extremely accurate depth judgments are required. Our performance results for Participant 10 did not support this hypothesis, and may indicate inflexibility in users' self-modification of depth-cueweightings. However, research suggests that at least for some pseudo-stereoanomalous viewers, practice or training on the use of disparity cues may help a viewer overcome such a deficient strategy. For instance, Fujisaki, Yamashita, Kihara, \& Ohtsuka (2012) reported that many pseudo-stereoanomalous viewers benefited from specific stereo training to help estimate object depths, presumably by learning to more heavily weight the available disparity cues. A follow-up study confirmed that all pseudo-anomalous viewers could use disparity to improve depth estimation (Fujisaki, Yamashita, Kihara, \& Ohtsuka, 2013). Unfortunately, our work did not touch on this interesting possibility of improving pseudo-stereoanomalous viewers' use of S3D via training, but this seems an area ripe for future research.

Participant 10 had demonstrated particular problems with uncrossed disparities, which harmed performance on the task. This participant had a relatively small fusion range (both near, far, and 
as tested on the S3D display), but these measures were not the smallest of the group. Even after follow-up testing, it is not clear why this participant would have had unique difficulty with uncrossed disparities or whether it is related in some manner to the phenomenon of stereoanomaly, which is typically demonstrated on time-limited detection or magnitude estimation tasks.

The explanation for Participant 7's results seems to be altogether different. Again, this participant tested normal on the clinical stereopsis measures, and had a decent stereoacuity of 2530 arc sec. But when this participant was tested using S3D displays, perceptual difficulty was reported, and performance was poor. On the placement task, whenever any disparity cues were present, performance declined relative to the zero-disparity session (except perhaps for stimuli located at the plane of the display). These results seem more consistent with the problems reported by Hoffman, Girshick, Akeley, and Banks (2008), in which a large subset of participants had clinically "normal" stereovision for real-world stimuli across a wide range of distances and disparities but had "problems with the experimental stimuli in which vergencefocal conflicts were present” (p. 21). Our results seem to support this interpretation of the V-A conflict being the culprit, given that stimuli with any disparities (crossed or uncrossed) taking the target off the plane of the display resulted in large performance deficits for Participant 7 . It is of note, too, that Participant 7 had small fusion ranges as measured clinically and when measured on the S3D display (in both instances, this Participant scored the 2nd smallest fusion ranges out of the 12 participants).

In light of these optometric tests and performance data on the placement task, the results suggest that Participant 7 may have had great difficulty in 'stretching' or otherwise modifying the reflexive neurological link between vergence and accommodation, thus making it difficult or perhaps impossible for this viewer to effectively view stereo imagery that requires vergence eye movements off the plane of the display (crossed or uncrossed disparities). This pattern of results may also be related to the phenomenon of stereoanomaly, since it is consistent with the idea of a deficiency in crossed or uncrossed mechanisms relative to near-zero disparity. It may also be consistent with a deficiency in coarse versus fine stereopsis mechanisms, or even consistent with the idea of vergence-anomaly as contributing to stereoanomaly (e.g., conflicting V-A depth cues could be confusing the eye movement system that is responsible for bringing disparate stimuli into alignment on the two retinas).

\section{Conclusions and Future Research}

In conclusion, we have demonstrated large individual differences in the performance benefits conferred by an S3D display using a virtual object precision placement task in depth, despite all participants being classifiable as possessing "normal” binocular and stereoscopic vision. Across the binocular disparity limit manipulations, one participant showed no general benefit to having S3D cues, although this viewer benefited from larger disparities yet was harmed by smaller disparities, and seemed to have particular difficulties with uncrossed disparities. And another participant found S3D disparity cues of any magnitude to be detrimental to performance, especially if the stimuli appeared off the plane of the display surface. 
What is particularly disturbing from an applied point-of-view is that all participants passed a standard clinical test for stereovision (the Titmus stereotest), demonstrated normal phorias and binocular fusion ranges, and all had stereoacuities of approximately 25-30 arc min or better. One issue we discovered when measuring stereoacuity thresholds was that two participants had great difficulty with this task on an S3D display, although their stereoacuities could be estimated using standard clinical stereotests such as the Titmus, the Randot, and the OVT. These were Participants 5 and 7; recall that Participant 7 was classified as an atypical performer, and Participant 5 was classified as a normal performer but received the smallest benefit of S3D out of the normal group. A reason for this finding might be that the standard clinical stereotests typically require an 'odd-man-out' depth detection (e.g., which of three circles appears in depth on the Wirt circles portion?). In contrast, our custom threshold task on the S3D display required judgments of 'near' versus 'far' disparity for a virtual test rod relative to flanking reference rods (a 'disparity sign' or 'depth direction' task). It is possible that these two participants have problems with disparity sign detection that is dissociable from simple disparity detection (which is what is tested on most clinical stereovision tests). This possibility was suggested by Landers and Cormack (1997) as a potential contributing issue to stereoanomaly, and may be an area worth pursuing for future research.

The atypical performance of Participants 7 and 10 is consistent with various descriptions of stereoanomaly but suggest the phenomena may not be limited only to briefly-presented stimuli, and may actually manifest in performance decrements on lengthier precision depth tasks involving fine stereopsis on S3D displays. In addition, one set of atypical performance results is also consistent with descriptions of pseudo-stereoanomaly (individual differences in depth-cueweighting strategies). Finally, both participants' atypical performance results may also be related to the vergence-accommodation conflict that is inherent in S3D display systems. There are likely individual differences in the ability to modify the V-A reflex in the presence of V-A cue conflict, as is required for properly viewing some S3D stimuli. At present, it is unclear how these three issues interrelate, if indeed they do, and future research on these topics is recommended.

\section{Acknowledgments}

Special thanks to the United States Air Force School of Aerospace Medicine (USAFSAM) Operationally-based Visual Assessment (OBVA) laboratory for the use of their clinical optometric facilities, and for their assistance in the data collection, analysis, and interpretation (especially Alex Van Atta, who created the custom stereoacuity threshold program). This work was derived from the first author's PhD dissertation, completed in May 2014 at Wright State University's Department of Psychology, with the human subject data collected at the Air Force Research Laboratory, Battlespace Visualization Branch, Wright-Patterson AFB. Thanks to all the participants who volunteered to contribute their valuable time. 


\section{References}

Blake, R. \& Wilson, H. (2011). “Binocular Vision.” Vision Research, 51, pp. 754-770.

Fielder, A. R., \& Moseley, M. J. (1996). “Does stereopsis matter in humans?” Eye, 10, pp. 233-238.

Froner, B. (2011). "Stereoscopic 3D technologies for accurate depth tasks: A theoretical and empirical study." Durham thesis, Durham University. Available online at: http://etheses.dur.ac.uk/3324/

Fujisaki, H., Yamashita, H., Kihara, K., \& Ohtsuka, S. (2012). "Individual differences in the use of binocular and monocular depth cues in 3D-graphics environments.” SID 2012 Digest, pp. 1190-1193.

Fujisaki, H., Yamashita, H., Kihara, K., \& Ohtsuka, S. (2013). "Ratio of pseudostereoanomalous young adults and improvement of their stereopsis in 3D-graphics environments: Study for depth perception based on the use of disparity and shading cues.” ITE Transactions on Media Technology and Applications (MTA), 1(3), pp. 93-99.

Hoffman, D. M., Girshick, A. R., Akeley, K., \& Banks, M. S. (2008). "Vergenceaccommodation conflicts hinder visual performance and cause visual fatigue.” Journal of Vision, 8(3), pp. 1-30.

Jones, R. (1977). “Anomalies of disparity detection in the human visual system.” Journal of Physiology, 264, pp. 621-640.

Kihara, K., Fujisaki, H., Ohtsuka, S., Miyao, M., Shimamura, J., Arai, H., \& Taniguchi, Y. (2013). "Age differences in the use of binocular disparity and pictorial depth cues in 3Dgraphics environments.” SID 2013 Digest, pp. 501-504.

Kooi, F. L., Dekker, D., van Ee, R., \& Brouwer, A.-M. (2010). "Real 3D increases perceived depth over anaglyphs but does not cancel stereo-anomaly.” Displays, 31, pp. 132-138.

Kytö, M., Hakala, J., Oittinen, P., \& Häkkinen, J. (2012). "Effect of camera separation on the viewing experience of stereoscopic images.” Journal of Electronic Imaging, 21(1), p. 011011-1.

Landers, D. D., \& Cormack, L. K. (1997). “Asymmetries and errors in perception of depth from disparity suggest a multicomponent model of disparity processing." Perception \& Psychophysics, 59(2), pp. 219-231.

McIntire, J. P., Havig, P. R., \& Geiselman, E. (2012). "What is 3D good for? A review of human performance on stereoscopic 3D displays.” Proceedings of SPIE, 8383, 83830X.

McIntire, J. P., Havig, P. R., \& Geiselman, E. (2014). "Stereoscopic 3D displays and human performance: A comprehensive review.” Displays, 35, pp. 18-26. 
McIntire, J. P., Wright, S. T., Harrington, L. K., Havig, P. R., Watamaniuk, S. N. J., \& Heft, E. L. (2014). "Optometric measurements predict performance but not comfort on a virtual object placement task with a stereoscopic three-dimensional display." Optical Engineering, 53(6), Special Section On Human Vision; 061711.

McIntire, J. P., Wright, S. T., Harrington, L. K., Havig, P. R., Watamaniuk, S. N. J., \& Heft, E. L. (in preparation). "Microstereopsis is good, but orthostereopsis is better: Precision alignment task performance and viewer discomfort with a stereoscopic 3D display." Journal of Electronic Imaging.

McIntire, J. P., Wright, S. T., Harrington, L. K., Havig, P. R., Watamaniuk, S. N. J., \& Heft, E. L. (in preparation). "Binocular fusion ranges and stereoacuity predict positional and rotational task performance on a stereoscopic 3D display.” Journal of Display Technology.

Mustillo, P. (1985). "Binocular mechanisms mediating crossed and uncrossed stereopsis." Psychological Bulletin, 97(2), pp. 187-201.

Patterson, R. \& Fox, R. (1984). “The effect of testing method on stereoanomaly." Vision Research, 24(5), pp. 403-408.

Richards, W. (1970). “Stereopsis and stereoblindness.” Experimental Brain Research, 10, pp. 380-388.

Richards, W. (1971). “Anomalous stereoscopic depth perception.” Journal of the Optical Society of America, 61(3), pp. 410-414.

Rozhkova, G., I., \& Vasiljeva, N. N. (2010). “A computer-aided method for the evaluation of fusional reserves with objective control of fusion break.” Human Physiology, 36(3), pp. 364-366.

Shimono, K. (1984). "Evidence for the subsystems in stereopsis: fine and coarse stereopsis." Japanese Psychological Research, 26(3), pp. 168-172.

Tyler, C. (1990). “A stereoscopic view of visual processing streams.” Vision Research, 30(11), pp. 1877-1895.

van Ee, R. (2003). "Correlation between stereoanomaly and perceived depth when disparity and motion interact in binocular matching." Perception, 32(1), pp. 67-84.

van Ee, R., \& Richards, W. (2002). “A planar and a volumetric test for stereoanomaly.” Perception, 31, pp. 51-64.

Watson, A. B., \& Pelli, D. G. (1983). “QUEST: A Bayesian adaptive psychometric method.” Perception \& Psychophysics, 33(2), pp. 113-120. 